\title{
A Direct Comparison of Three Buckling-Based
}

\section{Methods to Measure the Elastic Modulus of}

\section{Nanobiocomposite Thin Films}

Taylor C. Stimpson ${ }^{1}$, Daniel A. Osorio ${ }^{2}$,Emily D. Cranston ${ }^{2,3 *}$, Jose M. Moran-Mirabal ${ }^{4 *}$

${ }^{1}$ Department of Chemical Engineering, McMaster University, 1280 Main Street West, Hamilton, ON L8S 4L7, Canada

${ }^{2}$ Department of Chemical and Biological Engineering, The University of British Columbia, 2360 East Mall, Vancouver, BC V6T 1Z3, Canada

${ }^{3}$ Department of Wood Science, The University of British Columbia, 2424 Main Mall, Vancouver, BC, V6T 1Z4, Canada, and Department of Chemical and Biological Engineering, The University of British Columbia, 2360 East Mall, Vancouver, BC V6T 1Z3, Canada

${ }^{4}$ Department of Chemistry and Chemical Biology, McMaster University, 1280 Main Street West, Hamilton, ON L8S 4M1, Canada

\section{KEYWORDS}

Mechanical properties, strain-induced buckling, thin films, layer-by-layer films, cellulose nanocrystals, polyethyleneimine 


\section{ABSTRACT}

To engineer tunable thin film materials, accurate measurement of their mechanical properties is crucial. However, characterizing the elastic modulus with current methods is particularly challenging for sub-micrometer thick films and hygroscopic materials because they are highly sensitive to environmental conditions and most methods require free-standing films which are difficult to prepare. In this work, we directly compared three buckling-based methods to determine the elastic moduli of supported thin films: 1) biaxial thermal shrinking, 2) uniaxial thermal shrinking, and 3) the mechanically compressed, strain-induced elastic buckling instability for mechanical measurements (SIEBIMM) method. Nanobiocomposite model films composed of cellulose nanocrystals (CNCs) and polyethyleneimine (PEI) were assembled using layer-by-layer deposition to control composition and thickness. The three buckling-based methods yielded the same trends and comparable values for the elastic moduli of each CNC-PEI film composition (ranging from $15-44 \mathrm{GPa}$, depending on film composition). This suggests that the methods are similarly effective for the quantification of thin film mechanical properties. Increasing the $\mathrm{CNC}$ content in the films statistically increased the modulus, however, increasing the PEI content did not lead to significant changes. The standard deviation of elastic moduli determined from SIEBIMM was 2-4 times larger than for thermal shrinking, likely due to extensive cracking and partial film delamination. In light of these results, biaxial thermal shrinking is recommended as the method of choice because it affords the simplest implementation and analysis and is the least sensitive to small deviations in the input parameter values, such as film thickness or substrate modulus. 


\section{INTRODUCTION}

Over the last 15 years, buckling-based methods have emerged as an alternative to measure the elastic modulus of thin films, which circumvent limitations of traditional approaches. ${ }^{1}$ Bucklingbased methods take advantage of wrinkles that emerge when a rigid film on a soft substrate is subjected to compressive stress; the wrinkle size is characteristic of the film thickness and elastic modulus. Common mechanical testing methods, such as tensile testing, and even more advanced methods, like nanoindentation, are widely used and can give good insight into mechanical properties. However, for films made of polymeric materials, traditional methods can be limited in their material requirements and resolution, ${ }^{2,3}$ and often require free-standing films with thicknesses in the micrometer range. Thin films (nanometers to several micrometers in thickness ${ }^{4}$ ) attached to a substrate are readily used in protective/optical coatings, sensors, membranes, photovoltaics and electronics, such that measuring their modulus on a substrate is highly relevant for their application. Bulk sample measurements may not be reflective of the properties of thin films due to interface effects, ${ }^{5}$ and nanoindentation can have significant substrate effects and be overly localized, which may not give true properties of the film as a whole. ${ }^{3}$ Buckling-based methods are an attractive alternative to quantify the elastic modulus of supported polymer or polymer nanocomposite thin films because they are simple and inexpensive to implement, and allow multiplexed measurements. ${ }^{6}$

Strain-induced elastic buckling instability for mechanical measurements (SIEBIMM) was the first reported buckling-based method to measure the elastic modulus of thin films. ${ }^{1,7}$ In this method, a thin film is deposited on the surface of a compliant substrate, such as polydimethylsiloxane (PDMS). Upon mechanical compression of the sample (strain $\leq 1 \%$ ), the supported film buckles to dissipate strain, forming periodic sinusoidal wrinkles. ${ }^{8}$ The wrinkle 
wavelength is used to calculate the film's elastic modulus $\left(E_{f}\right)$, under the conditions that the film is much thinner and stiffer than the underlying substrate thickness $_{\mathrm{f}} \ll$ thickness $_{\text {substrate }}, E_{f} \gg$ $E_{\text {substrate }}$. The SIEBIMM method has been used to characterize organic and inorganic thin films with thicknesses of tens of nanometers to a few micometers. ${ }^{1,6,9-15}$ A limitation of SIEBIMM, however, is that crack formation across the film is common, which can interfere with modulus measurement by locally releasing stress and changing the wrinkle wavelength. ${ }^{1,15}$ Additionally, for characterization of bio-based and other hygroscopic materials, SIEBIMM needs to be performed under carefully controlled temperature and relative humidity (RH) conditions. ${ }^{14}$

Thermal shrinking is a more recent buckling-based method to measure elastic moduli whereby a thin film is subjected to high strains arising from the shrinking of a shape-memory polymer substrate above its glass transition temperature (such as pre-stressed polystyrene). ${ }^{2,16} \mathrm{~A}$ thin film deposited onto the substrate buckles as the substrate shrinks ${ }^{2}$ and, due to the high compressive stress in all directions, the resulting wrinkles are randomly oriented and typically an order of magnitude smaller than those in SIEBIMM. Thermal shrinking can be performed on an unconstrained sample (biaxial shrinking) or by constraining the sample along one axis via a clamping device (uniaxial shrinking). Uniaxial thermal shrinking has been previously reported for surface patterning, ${ }^{17-19}$ and gold film modulus measurement, ${ }^{16}$ but not for modulus measurements of polymer materials. The biaxial thermal shrinking method gives reliable elastic modulus measurements that are independent of the humidity/hydration level in the films (essentially giving a modulus at $\sim 0 \% \mathrm{RH}$ ), even for highly hygroscopic materials. ${ }^{2}$ An additional advantage of the thermal shrinking method is that the films are irreversibly wrinkled (i.e., plastically deformed), which means that they can be easily characterized after shrinking without needing an external device to maintain the strain. As with SIEBIMM, thermal shrinking is practically limited to a 
thickness range for the supported films, which must be sufficiently thin to avoid cracking or delamination upon wrinkle formation. Previous reports have shown that thin polymer and metallic films with thickness in the tens to hundreds of nanometers, ${ }^{2,16,17}$ and softer materials such as hydrogels as thick as $5 \mu \mathrm{m},{ }^{18}$ were not prone to cracks or film delamination in thermal shrinking. The upper limit of film thickness to prevent cracking/delamination is strongly dependent on the fracture toughness of the material.

SIEBIMM and thermal shrinking methods have been used to measure the mechanical properties for an extensive range of materials but have been particularly useful for polymer-based films that are more challenging to measure by nanoindentation than metallic and inorganic coatings. For example, SIEBIMM has been demonstrated for single-component polymer films of polystyrene, ${ }^{1,10,20,21}$ and poly(methyl methacrylate), ${ }^{10}$ as well as layer-by-layer (LbL) films composed entirely of polyelectrolytes, ${ }^{10,22-24}$ or of polymers and nanoparticles ${ }^{13,25,26}$ where the uniformity, reproducibility, and tunability inherent to the LbL method is particularly useful. Biobased nanoparticles, such as cellulose nanocrystals (CNCs) or cellulose nanofibrils (CNFs) combined with polyethyleneimine (PEI) and poly(allylamine hydrochloride) (PAH) have also been studied using buckling-based methods. ${ }^{2,14,15,27} \mathrm{CNCs}$ and $\mathrm{CNFs}$ are anionic rod-shaped and fiberlike nanocelluloses, respectively, and PEI is a highly branched cationic polyelectrolyte; ${ }^{15}$ this combination in particular leads to homogeneous films where the thickness is easily controlled by the number of deposition steps (bilayers). Nanocellulose on its own has exceptionally good mechanical properties, with average axial and transverse moduli of 110-167 $\mathrm{GPa}^{28-30}$ and 10-50 $\mathrm{GPa}^{28,29}$ respectively, making it highly promising as a reinforcing agent in composites. ${ }^{28,31}$ However to date, its full reinforcing ability has not been realized; as such this is an interesting model system to study while working towards a better understanding of nanocellulose's potential. 
Reported elastic moduli for supported PEI-nanocellulose films range from 3.5 - $72 \mathrm{GPa}^{2,14}$ (for PEI-CNC films) and $1.5-17.2 \mathrm{GPa}^{15}$ (for PEI-CNF films) when measured using buckling methods. The range and discrepancy in values is due to the different methods used (SIEBIMM vs. thermal shrinking), humidity (which affects SIEBIMM modulus calculation by $\sim 0.3 \mathrm{GPa}$ per \% change in $\mathrm{RH}^{14}$ ), and the different ratios of cellulose and PEI in the layered films. However, to our knowledge, no side-by-side comparison of the elastic moduli obtained from these buckling-based methods has been done for any kind of films with identical compositions.

In this work, three buckling-based methods were directly compared and used to calculate the elastic modulus of nanobiocomposite thin films including: 1) biaxial (unconstrained) thermal shrinking, 2) uniaxial (constrained) thermal shrinking, and 3) SIEBIMM. The elastic moduli of three compositions of CNC-PEI LbL films with varying concentrations of CNCs and PEI were determined. The goal was to compare the accuracy and precision of each method for measuring elastic modulus changes as they relate to film composition. A sensitivity analysis was also performed, to evaluate the sensitivity of the calculation of elastic modulus to small deviations in the measured parameters, giving an indication of the robustness of the methods. We anticipate that the use of a simple method based on thin film buckling will be useful to characterize the mechanical performance of a broad range of nanomaterials and nanocomposite films for applications including photovoltaics, ${ }^{32-35}$ biosensors, ${ }^{35,36}$ flexible electronics, ${ }^{37}$ stimulicontrollable materials, ${ }^{38}$ and point-of-care diagnostic sensors. ${ }^{39,40}$ 


\section{EXPERIMENTAL SECTION}

Materials

Pre-stressed polystyrene (PS) shrink films (Graphix Shrink Film, Maple Heights, OH, USA) were used as substrates for layered polymer nanobiocomposite and gold films. Cellulose nanocrystals in sodium-salt form as a spray dried powder were kindly donated by CelluForce (CelluForce NCC, Batch 2015-009, Celluforce, CA). Polyethyleneimine (PEI, MW 750,000 g/mol, 50\% (w/v) in water) was purchased from Sigma-Aldrich (Oakville, ON, Canada) and solutions of 0.1 and $1 \mathrm{wt} \%$ were prepared in water. Polyallylamine hydrochloride (PAH, MW 120,000 - 200,000 g/mol) was purchased from Polysciences (Warrington, PA, USA) and prepared at a concentration of $1 \mathrm{wt} \%$ in water. Gold (99.999\% purity, LTS Chemical Inc., Chestnut Ridge, NY, USA) was used for calibration of elastic modulus equations. Si wafers used as substrates were purchased from University Wafers (N-type, $<100>$ orientation, University Wafers, South Boston, MA, USA) and cleaned with piranha solution $\left(3: 1\right.$ solution of $\left.\mathrm{H}_{2} \mathrm{SO}_{4}: \mathrm{H}_{2} \mathrm{O}_{2}\right)$. All aqueous solutions were made using ultrapure 18.2 M $\Omega$ cm water (Milli-Q A10 Purification System, Millipore, Etobicoke, ON, Canada).

CNCs derived from various sources and protocols can have different properties, affecting interactions between particles and other materials, as well as resulting composite material properties (including elastic modulus). ${ }^{41} \mathrm{CNCs}$ from CelluForce were produced from $64 \mathrm{wt} \%$ sulfuric acid hydrolysis of bleached Kraft pulp, after which CNCs were separated from sulfuric acid, neutralized to the sodium-salt form, and spray dried. ${ }^{42}$ CelluForce CNCs (from the same batch as those used in this study) were reported to have a sulfur content of $0.8 \mathrm{~g} / 100 \mathrm{~g} \mathrm{CNC}$ which corresponds to a sulfate half-ester content of $250 \mathrm{mmol} / \mathrm{kg} \mathrm{CNC}$; zeta potential of $-40 \mathrm{mV}$; average length of $180 \pm 90 \mathrm{~nm}$, cross-section of $6 \pm 2 \mathrm{~nm}$, and aspect ratio of 31 as measured by atomic 
force microscopy; and $89-90 \%$ crystallinity measured by x-ray diffraction. ${ }^{42}$ Powdered CNCs (as received) were redispersed in ultrapure water at concentrations of 0.03 and $3 \mathrm{wt} \%$ by first slowly adding $\mathrm{CNC}$ powder to a beaker of water and stirring for $>1 \mathrm{~h} \cdot{ }^{41}$ Once no visible aggregates were observed in the CNC suspension, CNCs were point probe sonicated (Sonifier 450, Branson Ultrasonics, Danbury, CT) for 3 rounds of $30 \mathrm{~s}$ intervals with 1 min cooling between rounds at $60 \%$ maximum amplitude (120 $\mathrm{W}$ input power), while immersed in an ice bath (to prevent temperature of suspension from exceeding $\left.60^{\circ} \mathrm{C}\right) .{ }^{41}$

Material Preparation

Polystyrene (PS) Substrate Preparation:

Pre-stressed PS sheets were cut into $2 \times 2 \mathrm{~cm}$ square and $2 \times 5 \mathrm{~cm}$ rectangular substrates using a Robo Pro CE5000-40-CRP cutter (ROBOPro CE5000-40-CRP, Graphtec America Inc., Irvine, CA, USA) with the CB15UB ceramic blade (Graphtec America Inc., Irvine, CA, USA) with parameters of 30,1 , and 1 , for force, quality, and speed, respectively. The substrates were cleaned in consecutive baths of isopropanol, ethanol, and ultrapure water for 5 min each under $70 \mathrm{rpm}$ of orbital agitation. The substrates were dried with compressed nitrogen and stored in sealed containers until further use.

Polydimethylsiloxane (PDMS) Substrate Preparation:

PDMS (SYLGARD ${ }^{\circledR} 184$ Silicone Elastomer Kit, Dow Corning Corporation, Midland, MI, USA) was prepared by mixing base and curing agent in a 10:1 mass ratio for 2 min with rigorous stirring to achieve a homogenous mixture. The mixture was placed in a desiccator under vacuum for 30 min to remove residual trapped air. The PDMS was then poured into metallic molds with 
silicon wafer bases, to produce $60 \times 9 \times 5 \mathrm{~mm}(1 \times \mathrm{w} \times \mathrm{h})$ rectangles and $20 \times 4 \mathrm{~mm}(\mathrm{~d} \times \mathrm{h})$ circles. The PDMS substrates were cured for over $48 \mathrm{~h}$ at ambient conditions before removing them from the molds. The rectangles were used as substrates for LbL film assembly and SIEBIMM experiments, while cylinders were used for compression testing to obtain accurate elastic moduli for each batch of PDMS substrates.

Nanobiocomposite Film Construction: Layer-by-Layer Assembly:

Layered nanobiocomposite films of different CNCs and PEI compositions were constructed using diffusion-limited solution dip coating with the LbL method. This method results in thin film materials with alternating layers of CNCs and PEI with controllable thickness based on the number of layers deposited. Films were deposited onto either PS or PDMS for buckling, or Si wafer substrates for thickness measurements as illustrated in Figure 1.

Clean PS, Si, and cured PDMS substrates were first treated using air plasma at $600 \mathrm{mTorr}$ and high power (30 W) for 3 min (PDC001 Expanded Plasma Cleaner, Harrick Plasma, Ithaca, NY, USA) to increase surface energy and improve wettability. The treated substrates were then immediately dipped into a $1 \mathrm{wt} \%$ solution of PAH for $15 \mathrm{~min}$ to act as an anchoring layer and improve adhesion between the LbL film and the substrate to prevent film lift-off. The samples were rinsed by dipping in ultrapure water for $10 \mathrm{~min}$ to remove any loosely bound polymer. Following deposition of the PAH layer, alternating layers of CNCs and PEI were deposited by sequentially dipping the sample in the CNC suspension ( 0.03 or $3 \mathrm{wt} \%)$, and the PEI solution $(0.1$ or $1 \mathrm{wt} \%$ ) for 15 min each. The film compositions are denoted as $\mathrm{CNC}_{\mathrm{X}}-\mathrm{PEI}$, where $\mathrm{X}$ and $\mathrm{Y}$ represent the $\mathrm{CNC}$ and PEI concentration, respectively. In between each layer, a 10-min rinsing step was performed by dipping the samples in ultrapure water to remove loosely bound polymer 
or CNCs, followed by drying with compressed nitrogen. This process was repeated until 3, 5, 7, and 10 bilayers were deposited, where 1 bilayer (bL) denotes one layer of CNCs and one layer of PEI. Samples were stored in petri dishes at ambient temperature.

Table 1. Summary of CNC-PEI film compositions and their notation.

Component

Concentrations

\begin{tabular}{l|l|l}
\hline Notation & $\begin{array}{l}\text { CNCs } \\
(\mathbf{w t} \%)\end{array}$ & $\begin{array}{l}\text { PEI } \\
(\mathbf{w t} \%)\end{array}$ \\
\hline $\mathrm{CNC}_{0.03}-\mathrm{PEI}_{0.1}$ & 0.03 & 0.1 \\
$\mathrm{CNC}_{3.0}-\mathrm{PEI}_{0.1}$ & 3.0 & 0.1 \\
$\mathrm{CNC}_{3.0}-\mathrm{PEI}_{1.0}$ & 3.0 & 1.0
\end{tabular}

Gold Film Preparation:

Gold films were used to calibrate the elastic modulus equations for thermal shrinking. Gold's known material properties were used to determine a scaling factor to relate film persistence length to wrinkle wavelength. Gold films of $125 \mathrm{~nm}$ thickness were deposited onto square and rectangular PS substrates for thermal shrinking using a Torr Compact Research Coater CRC-600 manual planar magnetron sputtering system (Torr International, New Windsor, NY, USA) using a deposition rate of $0.5 \AA / \mathrm{s}$. Samples were stored in petri dishes at ambient conditions until further use.

Buckling-Based Methods:

Films were buckled using three different methods (biaxial shrinking, uniaxial shrinking, SIEBIMM) to determine the elastic moduli of LbL CNC-PEI films and assess the viability of using 
each method for thin film mechanical property measurements. Gold films were buckled for calibration using all three methods. Films for thermal buckling were deposited onto PS, whereas films for SIEBIMM were deposited onto PDMS. ${ }^{1}$ In both biaxial and uniaxial thermal shrinking, films deposited onto PS were placed in an oven for $15 \mathrm{~min}$ at $135^{\circ} \mathrm{C}$ (above the glass transition temperature of PS), causing the PS to shrink to $40 \%$ (by width) of its original size.

In biaxial thermal shrinking, the square sample was left fully unconstrained, allowing it to shrink in all directions, leading to randomly oriented wrinkles across the sample. In uniaxial thermal shrinking, the rectangular samples were clamped on opposite ends of the sample, constraining them along one axis, which produced aligned wrinkles as sample shrinking only occurred perpendicular to the clamping axis. ${ }^{17}$ PDMS was placed between the sample and the metal clamp for uniaxial shrinking to avoid sample slippage during shrinking. Once buckled and removed from the oven, the uniaxial samples were cooled before removing them from the clamps. SIEBIMM samples on PDMS were clamped in a homemade strain device (shown by Kan et al. ${ }^{14}$ ) and compressed, or strained, along one axis by up to $1 \%$. To carry out measurements at low $\mathrm{RH}$, the SIEBIMM method was performed in a glove bag (Aldrich ${ }^{\circledR}$ AtmosBag Z530220, Sigma Aldrich, Oakville, ON, CA) pressurized using compressed nitrogen gas with an additional crystallization dish containing desiccant placed within the bag.

\section{Characterization Methods}

Scanning Electron Microscopy (SEM):

Buckled films on PS were prepared for SEM imaging by mounting the samples to 1" stainless steel stubs using carbon tape and painting the edges with nickel paint to establish contact between the stub and sample, as well as reduce charging during imaging. Samples were coated with $7 \mathrm{~nm}$ 
of platinum using a Precision Etching Coating System (Model 682, Gatan Inc., CA, USA) to make the samples conductive and improve SEM image acquisition. Images were taken using a JEOL 7000F SEM (JEOL, Tokyo, Japan) at an acceleration voltage of $2 \mathrm{kV}$ and working distances between 3 and $6 \mathrm{~mm}$.

Variable Angle Spectroscopic Ellipsometry (VASE):

Thickness measurements of $\mathrm{LbL}$ films deposited on $\mathrm{Si}$ wafers were obtained using an

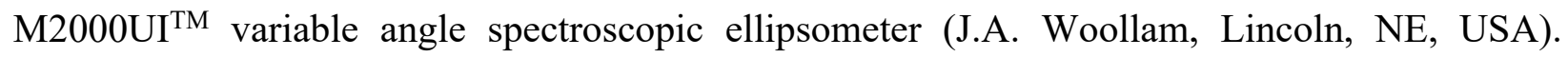
Measurements were taken over a range of wavelengths from $250-1680 \mathrm{~nm}$ and incident angles of 55 - 75 degrees, in 5-degree increments. The CompleteEASE® software was used to fit the ellipsometric data to a Cauchy model under the assumption of transparent films on an Si substrate in order to extract film thickness. Parameters fit for the model were confined to positive values.

\section{Light Microscopy:}

The optical microscope was placed in the nitrogen-filled glove bag to obtain images of SIEBIMM samples buckled in a low humidity environment. Images were taken using a BX51 upright microscope (Olympus, Center Valley, PA, USA) equipped with 4x, 10x, and 100x objectives, and a RETIGA 2000R CCD camera (Q Imaging, Surrey, BC, Canada).

\section{Compression Testing for Modulus Measurement:}

The cylindrical PDMS samples $(20 \times 4 \mathrm{~mm}, \mathrm{~d} \times \mathrm{h})$ were characterized by compression testing using an Instron mechanical tester (Model 3360 Universal Testing System, Instron, Norwood, MA, USA) following the ASTM D1229-03 standard testing method. A $5 \mathrm{kN}$ load cell and crosshead 
speed of $1.5 \mathrm{~mm} / \mathrm{min}$ were used to measure the elastic modulus of each batch of PDMS. The PDMS substrate elastic moduli $(1.9-2.2 \mathrm{MPa})$ were used in the calculation of thin film elastic moduli.

\section{Digital Image Processing \& Analysis:}

Images of buckled films taken at different magnitudes were cropped to $900 \times 900$ pixels and adjusted for contrast and brightness to improve feature detection, followed by Canny edge detection to detect wrinkle edges ${ }^{43}$ using ImageJ (ImageJ 1.52a, Wayne Rasband, National Institutes of Health). Fourier analysis and associated data filtering and curve fitting were performed using an algorithm developed in-house in MATLAB (MATLAB R2014b, MathWorks). ${ }^{44}$ This algorithm was optimized in previous work to accurately extract wrinkle wavelengths of films buckled using thermal shrinking and SIEBIMM and eliminate user bias. Power spectral density (PSD) plots obtained from Fourier transforms were averaged over the full range of angles for images of biaxially structured samples and from $75-105^{\circ}$ for uniaxial or SIEBIMM samples. All image analysis steps were performed, on images obtained from three replicate samples, for all CNC-PEI films over the range of thickness values buckled via all three methods, and gold films buckled using thermal methods.

\section{Calculation of Elastic Moduli:}

Plots of characteristic wavelength $(\lambda)$ as a function of film thickness $(h)$ were used to determine elastic moduli of LbL nanobiocomposite thin films. With the SIEBIMM method, Equation 1 was used to calculate the elastic modulus of the supported thin film: ${ }^{1}$

$$
E_{f}=3 E_{S} \frac{\left(1-v_{f}^{2}\right)}{\left(1-v_{S}^{2}\right)}\left(\frac{\lambda}{2 \pi h}\right)^{3}
$$


Where $E_{f}$ is the elastic modulus of the film, $E_{S}$ is the elastic modulus of the substrate, $v_{f}$ and $v_{S}$ are the Poisson's ratios for the film and substrate, respectively. The parameter $\lambda / h$ is extracted from the slope of a linear regression fit to a plot of characteristic wavelengths obtained from SIEBIMM over a range of film thickness values.

For both biaxial and uniaxial thermal shrinking, the film undergoes plastic deformation and high strain, in contrast to the elastic deformation and low strain of the SIEBIMM method. Therefore, there is a slight variation in the equation used to calculate the elastic modulus, as well as a scaling factor $(a)$ to relate wrinkle wavelength measured to the persistence length $(\xi)$. Data were fit to a linear regression, following the form:

$$
\xi=a \lambda=2 \pi \eta^{2 / 3} h
$$

Where Equation 2 was used to determine the factor $\eta$, which was in turn used to calculate elastic moduli following Equation 3:

$$
E_{f}=3 E_{S} \frac{\left(1-v_{f}^{2}\right)}{\left(1-v_{S}^{2}\right)} \eta
$$

All variables are the same as those in Equation 1 for SIEBIMM, however, in thermal shrinking, $E_{S}$ and $v_{S}$ are for the substrate at the processing temperature, in this case PS at $135{ }^{\circ} \mathrm{C}\left(v_{S}=0.36\right.$ and $\left.E_{S}=1.63 \mathrm{GPa}\right) .{ }^{45}$ The scaling factor $(a)$ was calibrated by measuring characteristic wavelengths of thin films of sputtered gold with a known thickness and reported Poisson's ratio $(0.42)^{46}$ and elastic modulus (70 GPa) ${ }^{16,46}$ For the film, $v_{f}=0.3$ was used, which is in between the values reported for CNCs $(0.28)$ and PEI (0.33) alone. ${ }^{47}$

Sensitivity Analysis:

A sensitivity analysis based on the one-at-a-time method ${ }^{48}$ was performed to assess how small deviations in the values of measured variables affected the calculated elastic moduli for the three 
buckling methods. All sensitivity analyses were performed using the "base case" of $\mathrm{CNC}_{3.0}-\mathrm{PEI}_{0.1}$ films. The ratio of the elastic modulus calculated from changed parameters was divided by the baseline elastic modulus $\left(E / E_{0}\right)$ and plotted as a function of changes in individual variables. Specifically, the wavelength $(\lambda)$ and film thickness $(h)$ were varied for all methods. The scaling factor $(a)$ and the elastic modulus of the PDMS $\left(E_{s}\right)$ were varied for biaxial/uniaxial shrinking and SIEBIMM, respectively. All variables were changed over a range of $-2 \sigma$ to $+2 \sigma$, where $\sigma$ was the standard deviation in each measured variable, and the elastic modulus was calculated with those new values, ceteris paribus.

\section{RESULTS AND DISCUSSION}

\section{Buildup and Characterization of LbL Films}

LbL films made of CNCs and PEI were deposited onto pre-stressed PS, PDMS, and Si wafers for thermal shrinking, SIEBIMM, and thickness measurements, respectively. The films were fabricated using different concentrations of CNCs $(0.03$ or $3 \mathrm{wt} \%)$ and PEI ( 0.1 or $1 \mathrm{wt} \%)$ (Table 1), to examine how changes in the film composition would be reflected in the moduli obtained from the three buckling-based methods. Layer build up in these films is thermodynamically controlled $^{49}$ and driven by electrostatic interactions between alternating layers of anionic $\mathrm{CNCs}$

and cationic PEI polymer chains. ${ }^{50}$ Schematic representations of the dip coating process and layer buildup are shown in Figure 1. 

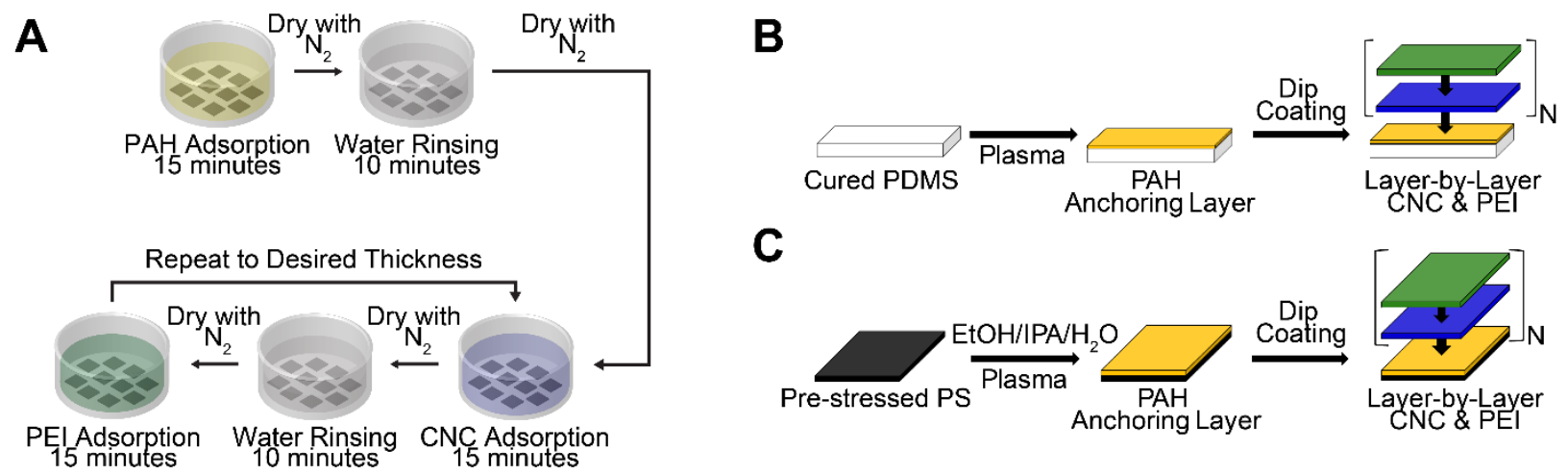

Figure 1. Schematic representation of A) the aqueous dip coating process of CNC-PEI nanobiocomposite films, and the LbL deposition of films on B) PDMS, and C) pre-stressed PS.

The thickness of dry CNC-PEI LbL films was measured by ellipsometry to examine the evolution of the film thickness as a function of the number of layers deposited (Figure 2). For all films, linear growth was observed. Linear regression analysis of film thickness versus bilayer number yielded slope values of $6.3 \pm 0.1\left(\mathrm{CNC}_{0.03}-\mathrm{PEI}_{0.1}\right), 21.5 \pm 0.6\left(\mathrm{CNC}_{3.0}-\mathrm{PEI}_{0.1}\right)$, and $26 \pm 1$ $\mathrm{nm} /$ bilayer $\left(\mathrm{CNC}_{3.0}-\mathrm{PEI}_{1.0}\right)$. This indicates that the films have significantly different compositions with thickness increasing with higher concentration dipping solution/suspensions. It was also noted that $\mathrm{CNC}$ concentration affects film thickness more than PEI concentration, which is expected since CNCs have been shown to provide the bulk of the thickness of composite LbL films. ${ }^{2}$ The thickness increment per bilayer for films constructed from $3 \mathrm{wt} \% \mathrm{CNCs}$ also agrees with those reported by Gill et al. ${ }^{2}$ who measured $19 \pm 1$ and $28 \pm 1 \mathrm{~nm} /$ bilayer for $\mathrm{CNC}_{3.0}-\mathrm{PEI}_{0.1}$ and $\mathrm{CNC}_{3.0}-\mathrm{PEI}_{1.0}$, showcasing the reproducibility of the coating method. 


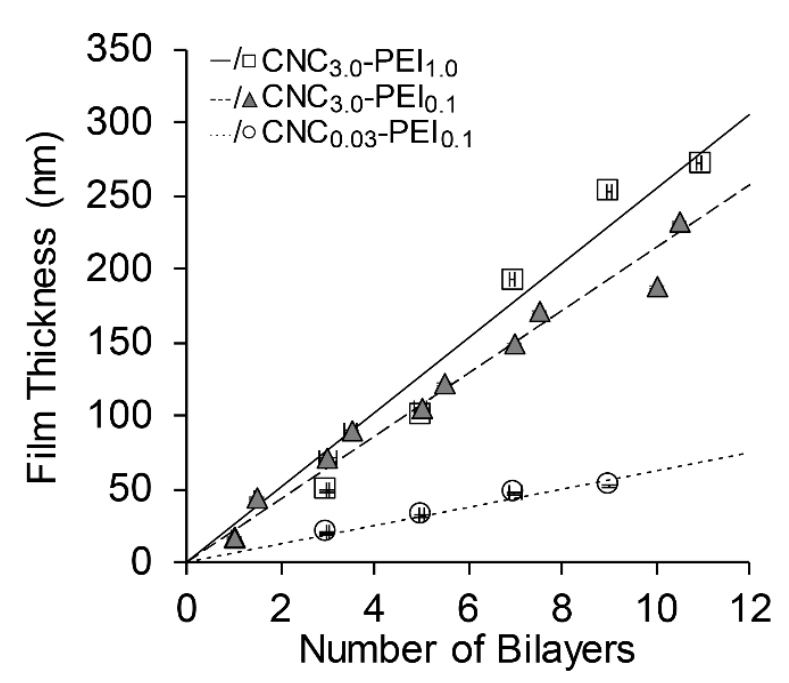

Figure 2. Plot of dry film thickness of CNC-PEI nanobiocomposite films as a function of the number of bilayers deposited. Error bars represent the standard deviation from $\mathrm{n} \geq 3$ measurements from 3 replicate samples and are smaller than the data symbols used.

CNC-PEI films have highly reproducible and controllable film thickness when made using LbL deposition and are therefore useful for this method comparison study. Given that the cross section of $\mathrm{CNCs}$ used here is approximately $6 \mathrm{~nm},{ }^{42}$ we conclude that films made with low CNC concentration have a single layer of CNCs and PEI deposited. However, for films made with a high concentration of CNCs, a double layer (or more) of the nanoparticles is inferred, which also concurs with previous reports that LbL buildup at high CNC concentrations leads to the adsorption of multiple nanoparticle layers. ${ }^{51-53}$ Nonetheless, for all film compositions it is assumed that the thickness of one bilayer reflects the buildup of at least one layer of CNCs and PEI. The highly branched nature of PEI can assist in the adsorption of multiple CNC layers in one deposition step and also suggests that intermixing between layers occurs and the films are not strictly "stratified" in discrete layers; this means that the internal film morphology is more similar to nanocomposites processed in bulk than some LbL films described in the literature. ${ }^{12,52}$ 


\section{Buckling of CNC-PEI Layered Thin Films}

Thermal shrinking and SIEBIMM methods were used to buckle CNC-PEI films to evaluate their elastic modulus. Films on PS were buckled either unconstrained (biaxial shrinking, Figure 3A) or constrained along one axis (uniaxial shrinking, Figure 3B), whereas films deposited onto PDMS were buckled by compressing the substrate mechanically (SIEBIMM method, Figure 3C). The buckled films were later imaged and image analysis was used to extract wrinkle information that allowed us to calculate the elastic moduli of the films as described below. Thus, these buckling methods facilitate the characterization of the mechanical properties of supported thin films, circumventing limitations of other mechanical testing methods such as nanoindentation or tensile testing.

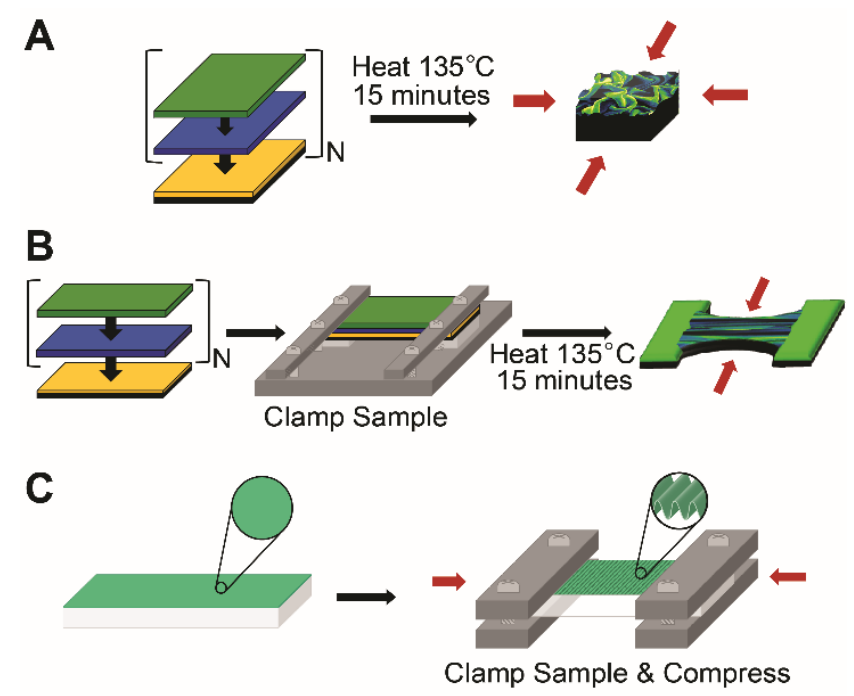

Figure 3. Schematic representation of buckling methods and their respective conditions for A) biaxial thermal shrinking, B) uniaxial thermal shrinking, and C) SIEBIMM. 
For thermally induced buckling, films were shrunk at $135^{\circ} \mathrm{C}$ (above the glass transition temperature of the PS substrate) causing the supported thin film to wrinkle based on the filmsubstrate elastic modulus mismatch. After thermal shrinking and cooling, the films remain buckled and, because the wrinkle size does not change with humidity or degree of film hydration prior to or post heating, ${ }^{2}$ the permanently wrinkled films can be characterized under any conditions. Shrinking at $135^{\circ} \mathrm{C}$ is not expected to affect CNCs, as their thermal degradation is significantly above this temperature, ${ }^{54}$ and because the glass transition temperature of PEI is so low (-60 to -50 $\left.{ }^{\circ} \mathrm{C}\right),{ }^{55,56}$ that it is in the same "rubber" state at both ambient temperature and during shrinking. Additionally, PEI is not expected to degrade during shrinking, as it has been shown that the onset of degradation occurs around $155^{\circ} \mathrm{C} .{ }^{57}$ Thermally buckled CNC-PEI films were imaged using SEM since their wrinkle sizes were in the sub-micrometer to micrometer range (Figure 4A and 4B). A higher magnification SEM image of a representative $\mathrm{CNC}_{3.0}-\mathrm{PEI}_{0.1}$ film, buckled via biaxial thermal shrinking, can be found in Figure S1 (Supporting Information), where the full coverage of CNCs across the film can be more clearly observed.

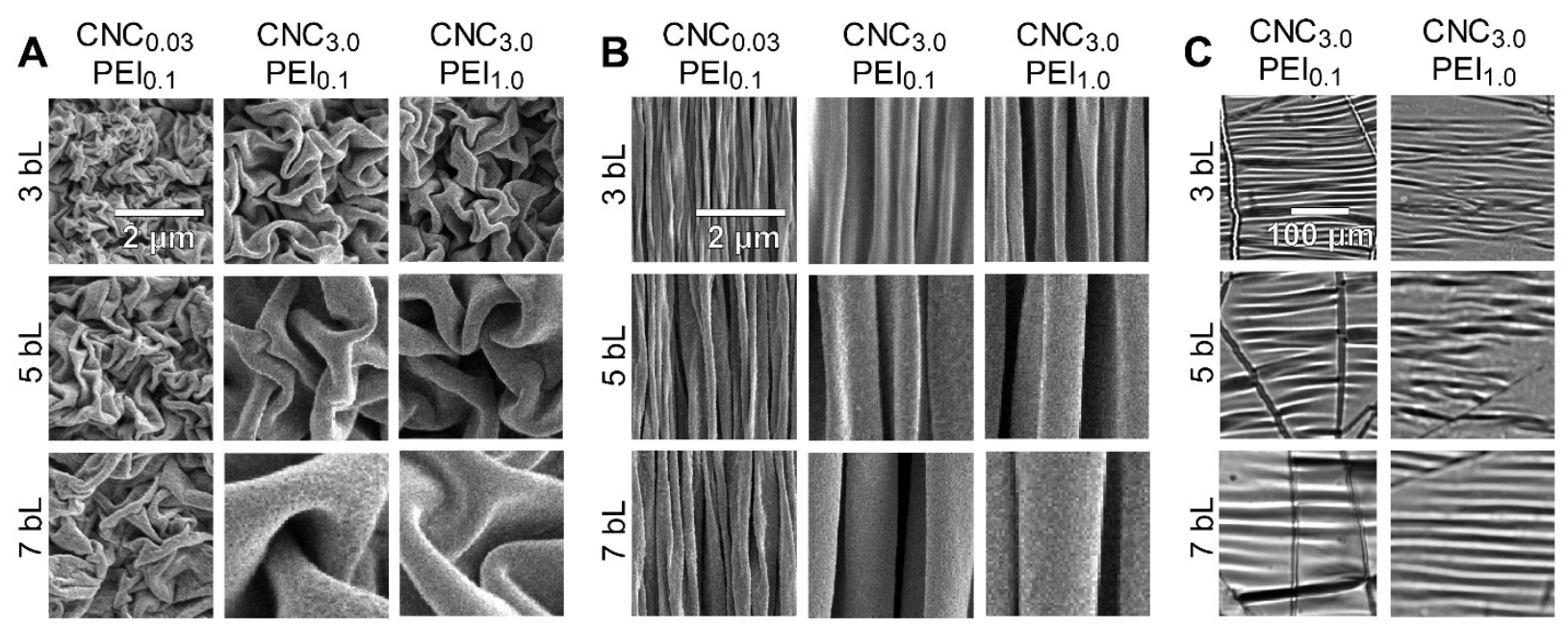


Figure 4. Scanning electron microscopy images of nanobiocomposite CNC-PEI films of different thicknesses (3, 5, and 7 bilayers, bL) and compositions buckled using A) biaxial thermal shrinking, B) uniaxial thermal shrinking, and C) SIEBIMM methods.

For the SIEBIMM method, the compliant PDMS substrate was compressed with very small strains $(<1 \%)$ compared to thermal shrinking ( $\sim 60 \%$ strain along each axis), and the film on the surface buckles into a sine wave pattern. Wrinkles occur along the axis in which the strain is applied. Because CNCs and PEI are very hygroscopic, changes in humidity can affect the thickness and modulus of the films, altering the wrinkle wavelength. To circumvent these effects, SIEBIMM was performed under a nitrogen atmosphere to minimize the relative humidity during buckling, and to mimic the (dry) thermal shrinking environment; this allowed us to directly compare the mechanical moduli calculated from the different methods. Since SIEBIMM compression is a reversible elastic deformation and the wrinkles were in the tens of micrometer range (for higher CNC concentrations), the samples were directly imaged using optical microscopy (Figure 4C), while maintaining compression in the strain device.

For all CNC-PEI compositions, wrinkle size increased with film thickness (Figure 4: 3, 5, and 7 bL buckled films from top to bottom), but the topographies differed between buckling methods. For biaxially buckled films (Figure 4A), the wrinkles were uniform across the entire sample with no preferred orientation. The unconstrained PS shrinks isotropically in the transverse dimensions, and the adhered film dissipates strain by buckling in all directions. Due to the high strain, multilevel structuring occurs leading to wrinkles on top of wrinkles. In uniaxial shrinking, the PS is clamped in one axis, which allows shrinking only in one direction and results in buckles that align with the free axis (Figure 4B). ${ }^{16-18}$ In contrast, films buckled via SIEBIMM (Figure 4C) have a 
highly regular sinusoidal topology. However, cracks appeared for all films perpendicular to the wrinkles due to the expansion of the film in the opposite direction to compression (characteristic of positive Poisson's ratio materials), which is a common challenge for this method. ${ }^{1}$ To account for the extensive cracking, more images were taken for analysis in hopes of averaging out any inconsistencies due to cracking. Unfortunately, SIEBIMM data for the thinnest $\mathrm{CNC}_{0.03}-\mathrm{PEI}_{0.1}$ films could not be collected because the wrinkles were smaller than could be imaged with the microscope objectives and working distance available (since the strain device needed to fit on the microscope stage).

Qualitatively, all three buckling methods show that $\mathrm{CNC}$ concentration during film assembly impacts the mechanical properties of the films much more than the PEI concentration. This can be seen from the fact that the wrinkle sizes for $\mathrm{CNC}_{3.0}-\mathrm{PEI}_{0.1}$ films are very similar to those obtained from $\mathrm{CNC}_{3.0}-\mathrm{PEI}_{1.0}$ in all methods. On the other hand, the wrinkle sizes for $\mathrm{CNC}_{3.0}-\mathrm{PEI}_{0.1}$ films are drastically larger than those obtained in $\mathrm{CNC}_{0.03}-\mathrm{PEI}_{0.1}$ films, indicating a higher modulus for films containing more CNCs. This confirms the observation made previously that in CNC-polymer thin films, CNCs have a greater contribution to the overall film thickness and mechanical properties than the polymer, ${ }^{14,57,58}$ and account for the bulk of the rigidity in the films. ${ }^{2}$ The contribution of $\mathrm{CNCs}$ to overall mechanical properties has also been explored in literature for bulk composites; ${ }^{28,58,59}$ in general, if CNCs are well dispersed they improve the tensile strength and elastic modulus of a polymer by up to $500 \%^{28,58}$ and $86 \%{ }^{60,61}$, respectively. Small loadings $(<10$ $w t \%)$ are often sufficient to greatly impact mechanical properties. For example, $7.5 \mathrm{wt} \% \mathrm{CNCs}$ in polyethylene oxide (another hydrophilic, low glass transition temperature polymer like PEI) resulted in a $60 \%$ increase in elastic modulus compared to the polymer without CNCs. ${ }^{60}$ Based on 
previous measurements, we expect that these films are composed of $>75 \% \mathrm{CNCs}^{2}$ and therefore large increases in modulus over PEI are expected.

Comparison of the Elastic Moduli Determined from Buckling Methods

The elastic moduli of CNC-PEI films calculated from biaxial/uniaxial thermal shrinking and SIEBIMM were directly compared. A Fourier analysis routine was used to obtain accurate wrinkle sizes from microscopy images ${ }^{44}$ which is an improvement over the weighted average of peaks used in previous Fourier analyses for identifying characteristic wavelengths of wrinkled films, ${ }^{2}$ or manual measurements of selected wrinkles. ${ }^{15}$ More specifically, the routine was optimized for high throughput, unbiased, and accurate measurement of periodic surface features. The raw images were first converted into binary edge maps using a Canny edge detection algorithm; a discrete 2D fast Fourier transform of the edge maps yielded PSD plots, which were then fitted to a Gaussian curve to obtain the characteristic spatial frequency. The characteristic wavelength was finally calculated as the inverse of this spatial frequency. An example of this analysis workflow is shown for $\mathrm{CNC}_{3.0}-\mathrm{PEI}_{0.1}$ films with 5 bilayers buckled using biaxial shrinking (Figure 5A), uniaxial shrinking (Figure 5B), and SIEBIMM methods (Figure 5C). 

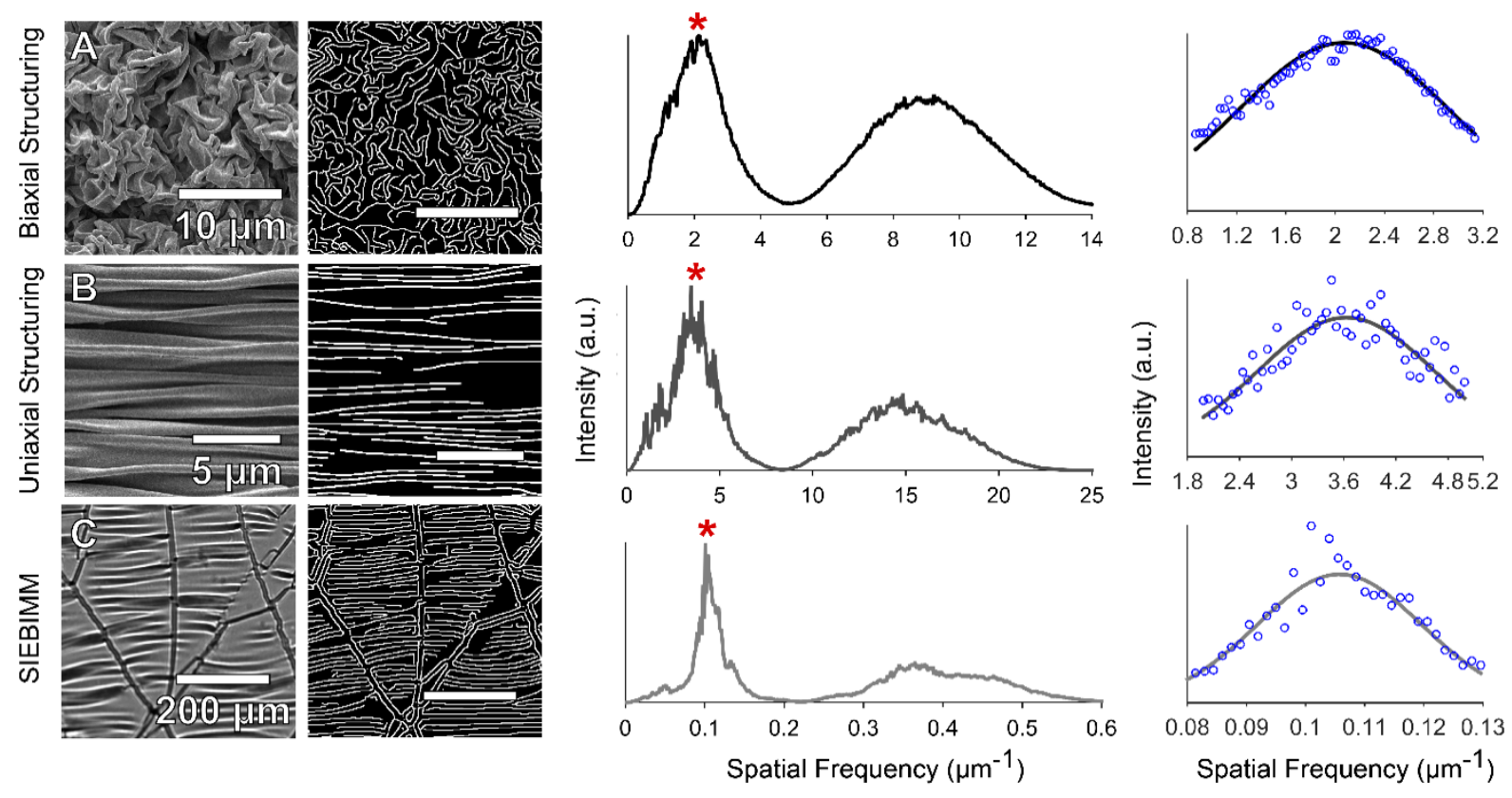

Figure 5. Scanning electron microscopy images of A) biaxial thermal shrinking, B) uniaxial thermal shrinking, and optical microscopy image of C) SIEBIMM of $\mathrm{CNC}_{3.0}-\mathrm{PEI}_{0.1}$ films with 5 bilayers shown with (left to right) the respective binary edge maps, 2D Fast Fourier transform power spectral density plots, and Gaussian curve fit of the relevant spatial frequency peaks (identified with a red asterisk).

Characteristic wrinkle wavelengths could be reliably extracted using the Fourier analysis routine, even when the structures were not perfectly periodic across the entire sample, or when there were discontinuities in the periodicity. ${ }^{44}$ It should be noted that although broadening occurs in the spatial frequency peak of the PSD plots due to the dispersity in the size of the periodic features, a characteristic wrinkle wavelength can still be identified. This peak broadening effect increases from SIEBIMM, to uniaxial shrinking, to biaxial shrinking as the wrinkles become more randomly oriented. In addition, since the PSD plots from the Fourier analysis were averaged over a restricted range of angles for samples with aligned features, the cracks in the films buckled via 
the SIEBIMM method (Figure 5C) did not interfere the process of determining the feature size. The characteristic wavelengths for films with different CNC-PEI compositions were plotted as a function of film thickness for all three methods (Figure 6). The measured wavelengths increased in the order $\mathrm{CNC}_{0.03}-\mathrm{PEI}_{0.1}<\mathrm{CNC}_{3.0}-\mathrm{PEI}_{0.1}<\mathrm{CNC}_{3.0}-\mathrm{PEI}_{1.0}$ (i.e., with increasing bilayer thickness) and for biaxial < uniaxial < SIEBIMM methods. Slope values from Figure 6 and the calculated moduli are summarized in Table 2.
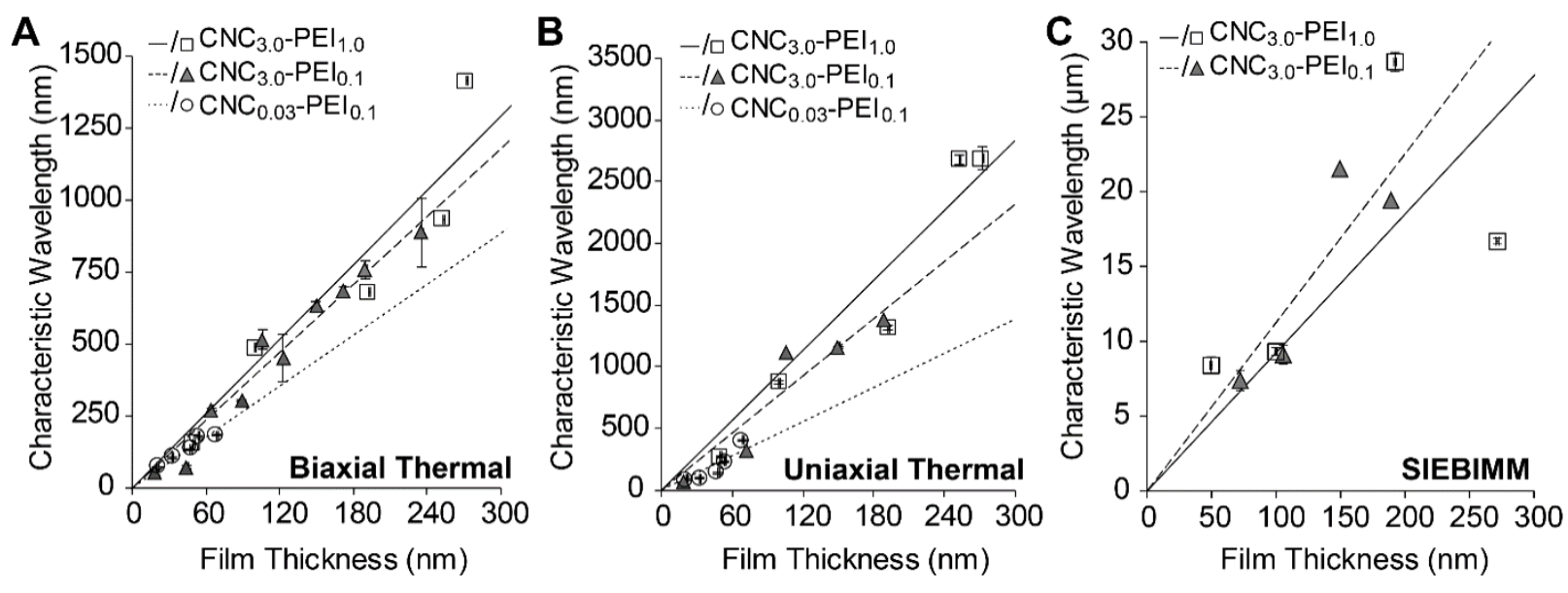

Figure 6. Plots of characteristic wavelengths as a function of film thickness for CNC-PEI nanobiocomposite films buckled using the A) biaxial thermal shrinking, B) uniaxial thermal shrinking, and C) SIEBIMM methods. Error bars represent the standard deviation from $\mathrm{n} \geq 3$ sample replicates. All linear regressions were forced to go through the origin and have $\mathrm{R}^{2}>0.96$ for thermal shrinking and $\mathrm{R}^{2}>0.84$ for SIEBIMM.

Table 2. Slopes from linear regression analysis of wavelength versus film thickness data, and elastic moduli calculated for CNC-PEI films, buckled via all three methods. Errors reported are propagated from error in the fits. SIEBIMM wavelength data could not be collected for $\mathrm{CNC}_{0.03-}$ 
$\mathrm{PEI}_{0.1}$ films due to the lack of an available objective with sufficient magnification and a working distance that could fit between the clamps of the strain stage.

\begin{tabular}{cccccccc} 
& \multicolumn{3}{c}{ Slope } & & \multicolumn{3}{c}{ Elastic Modulus } \\
& \multicolumn{2}{c}{ (nm wavelength/nm thickness) } & & \multicolumn{3}{c}{ (GPa) } \\
\cline { 2 - 4 } $\begin{array}{c}\text { Film } \\
\text { Composition }\end{array}$ & $\begin{array}{c}\text { Biaxial } \\
\text { Thermal }\end{array}$ & $\begin{array}{c}\text { Uniaxial } \\
\text { Thermal }\end{array}$ & SIEBIMM & & $\begin{array}{c}\text { Biaxial } \\
\text { Thermal }\end{array}$ & $\begin{array}{c}\text { Uniaxial } \\
\text { Thermal }\end{array}$ & SIEBIMM \\
\hline $\mathrm{CNC}_{0.03}-\mathrm{PEI}_{0.1}$ & $3.0 \pm 0.2$ & $4.6 \pm 0.6$ & - & & $21 \pm 2$ & $15 \pm 3$ & \\
$\mathrm{CNC}_{3.0}-\mathrm{PEI}_{0.1}$ & $4.2 \pm 0.2$ & $7.7 \pm 0.7$ & $110 \pm 10$ & & $35 \pm 3$ & $33 \pm 5$ & $40 \pm 10$ \\
$\mathrm{CNC}_{3.0}-\mathrm{PEI}_{1.0}$ & $4.3 \pm 0.3$ & $9.4 \pm 0.7$ & $90 \pm 20$ & & $37 \pm 5$ & $44 \pm 5$ & $20 \pm 20$ \\
\hline
\end{tabular}

The elastic moduli calculated from the three buckling methods were similar and fell within the ranges expected for CNC-polymer composites, where moduli for CNCs and PEI have been reported as $57-143 \mathrm{GPa}^{29,62}$ and $0.3 \mathrm{GPa},{ }^{15}$ respectively. We observed that the moduli increased with increasing $\mathrm{CNC}$ content in the films and with increasing film thickness increments/bilayer (i.e., the slopes from Figure 2). Statistical testing revealed that for biaxial and uniaxial thermal shrinking methods, there is a significant difference $(\mathrm{P}<0.05)$ between the elastic moduli of the films when the $\mathrm{CNC}$ content is increased from 0.03 to $3 \%$, whereas there is no significant difference when PEI is increased from 0.1 to $1 \%$. This is expected, since CNCs have a higher elastic modulus and have been shown to contribute extensively to mechanical properties of CNCpolymer materials. ${ }^{60,63}$ As well, the CNC concentration was changed by 100 -fold, versus the $10-$ fold change in the polymer concentration. SIEBIMM showed no significant difference in moduli when PEI concentration was increased from 0.1 to $1 \mathrm{wt} \%$ (where the large associated standard deviation in modulus for $\mathrm{CNC}_{3.0}-\mathrm{PEI}_{1.0}$ is due to extensive cracking and partial film delamination). Furthermore, the elastic moduli for CNC-PEI films of any given composition are statistically 
equivalent $(95 \%$ confidence, as tested by paired t-test and ANOVA, Table S1 in Supporting Information) across the three buckling methods.

The data in Table 2 show that all three buckling-based methods are viable for characterizing the mechanical properties of substrate-supported nanobiocomposite thin films with sub-micrometer thickness. Biaxial and uniaxial thermal shrinking methods measured significant $(\mathrm{P}<0.05)$ changes in mechanical properties with changes in film composition when there were large differences in film thickness per bilayer $\left(\mathrm{CNC}_{0.03}-\mathrm{PEI}_{0.1}\right.$ versus $\mathrm{CNC}_{3.0}-\mathrm{PEI}_{0.1}$ and $\left.\mathrm{CNC}_{3.0}-\mathrm{PEI}_{1.0}\right)$. Only uniaxial thermal shrinking, however, gave significantly different moduli between film compositions with little difference in thickness per bilayers (confirmed via one-way ANOVA, with $\mathrm{P}<0.05$ and $\mathrm{F}>$ $\mathrm{F}_{\text {crit, }}$ Supporting Information Table S1). This is relevant for materials for which mechanical property characterization is challenging, which is the case for substrate-supported thin films and highly hygroscopic materials.

While all three methods can be used to characterize thin film elastic moduli, we emphasize that testing was only done on films $<350 \mathrm{~nm}$ thick (and the upper limit will depend on film modulus and method used) and caution that cracking or delamination can decrease the precision of the methods substantially. These results also support that moduli can be compared within a method, and for the first time, show that absolute moduli can be directly compared across methods for the same materials (given the measurements are performed under the same experimental conditions). However, thermal shrinking methods consistently give more reproducible values, with lower standard deviation between replicates than for SIEBIMM, benefiting from the lack of film cracking and delamination observed in SIEBIMM. 
Comparison and Sensitivity Analysis

To assess how small deviations in measured variables impact the calculated elastic moduli, sensitivity analyses were performed for all three buckling-based methods based on the one-at-atime approach. ${ }^{48}$ In the sensitivity analysis, the values measured in $\mathrm{CNC}_{3.0}-\mathrm{PEI}_{0.1}$ films for thickness $(h)$, characteristic wavelength $(\lambda)$, and scaling factor ( $a$, in the case of thermal shrinking) or substrate modulus $\left(E_{s}\right.$, in the case of SIEBIMM) were varied $\pm 2 \sigma$ around the mean and plotted against the relative modulus (Figure 7).
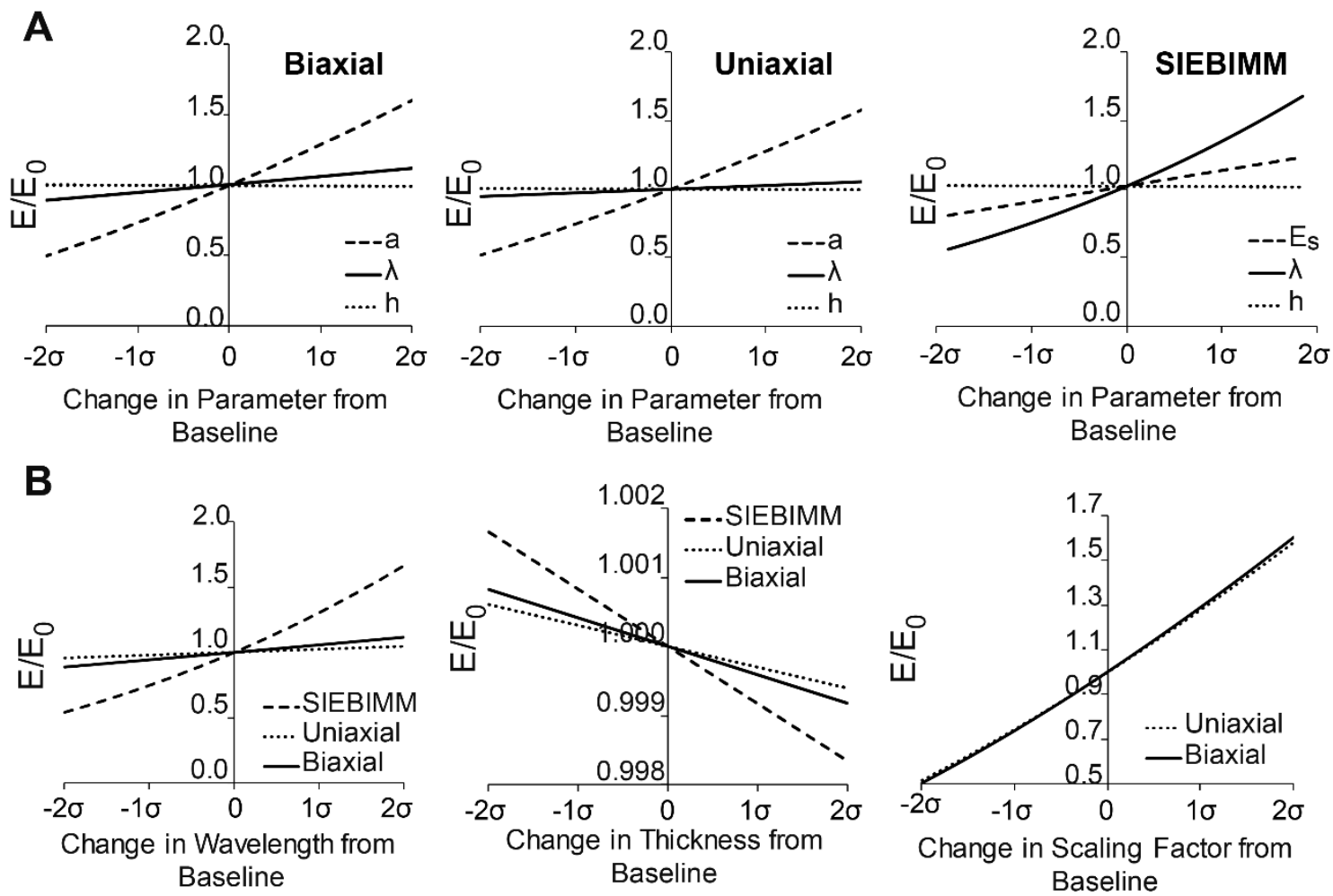

Figure 7. A) Sensitivity analysis plots for biaxial thermal shrinking, uniaxial thermal shrinking, and SIEBIMM methods. B) Sensitivity analysis plots comparing all buckling methods to their sensitivity to different variables: wrinkle wavelength $(\lambda)$, film thickness $(h)$, and scaling factor $(a)$. It should be noted that for the third plot in B) there is no plot of SIEBIMM since there is no scaling factor in Equation 1 for elastic modulus based on that method. The elastic modulus at each point, 
divided by the baseline modulus, is plotted as a function of changes in individual variables (as indicated by the legends) over a range of $-2 \sigma$ to $2 \sigma$, where $\sigma$ is the average standard deviation in each variable.

From the sensitivity analyses, it was determined that for thermal shrinking methods, the parameters that affect the value of the modulus are, in order from highest to lowest impact, the scaling factor $(a)$, characteristic wavelength $(\lambda)$, and film thickness $(h)$. That the scaling factor is the most sensitive parameter in the determination of the elastic modulus highlights the need for reliable buckled calibration films and robust methods to accurately measure the characteristic wavelength. These requirements justify the use of buckled gold calibration films and the recently developed Fourier analysis algorithm with curve fitting. ${ }^{44}$ In the case of SIEBIMM, the elastic modulus is impacted the most by changes in the wrinkle wavelength, followed by the elastic modulus of the PDMS substrate $\left(E_{s}\right)$, and the film thickness (Figure 7A). To ensure more reproducible measurements for SIEBIMM, a large number (at least 9) of repeat measurements of the wrinkle wavelength should be obtained since there can be variability in wrinkle size across the sample. As well, PDMS should be made under similar experimental conditions for each substrate, ensuring that the PDMS elastic modulus is measured for each batch of substrates produced. The film thickness seems to be of lowest sensitivity for all methods from this analysis, which can be attributed to a low error in measurements from spectroscopic ellipsometry. If other methods for measuring film thickness are used that have lower precision (larger $\sigma$ ), then it would likely result that the modulus would be more sensitive to changes in the film thickness than presented here. Based on this analysis, it is evident that all parameters need to be measured with minimal error to 
obtain the most reliable elastic moduli, but that the precision in measuring the characteristic wrinkle wavelength is key.

When comparing the sensitivity across methods, it was observed that the elastic moduli obtained from both thermal shrinking methods are similarly sensitive to the different variables assessed, while those obtained from SIEBIMM are much more sensitive to changes in the measured variables (Figure 7B). Based on this, we suggest that thermal shrinking methods are more robust methods for calculating elastic moduli, and afford larger tolerances for each measured variable, compared to SIEBIMM. Thermal methods are also attractive based on simpler methods for fabrication, buckling, and imaging, as well as being insensitive to environmental humidity conditions compared to SIEBIMM.

Based on results of sensitivity analyses and method requirements, equipment, and capabilities, biaxial thermal shrinking is the simplest and most robust method demonstrated here. The SIEBIMM method is the least precise of the three, requires the most equipment (strain device and method for humidity control), and is the most sensitive to minor variations in measured variables. However, SIEBIMM presents the opportunity to reversibly deform a sample, structure thermally sensitive samples (no heating is involved) or measure the effect of RH on film mechanical properties. Finally, since the SIEBIMM method wrinkles are on the order of micrometers, a standard light microscope can normally be used to image topography. This is considerably more accessible and inexpensive compared to an electron microscope used to image the nano-scale topography of thermally buckled samples. Conversely, biaxial thermal shrinking requires the least amount of equipment of the three methods (no clamp or strain device), gives similar modulus results to SIEBIMM and uniaxial shrinking with more precision than SIEBIMM, and is minimally sensitive to parameter variability or error. Overall, all three methods give reasonable and 
comparable elastic moduli in the range of tens of GPa, on films less than $350 \mathrm{~nm}$ thick, as shown with the polymer-nanoparticle nanobiocomposite films explored in this study. Thus, depending on material constraints or the goal of the research (precise measurement, humidity effects, surface structuring), methods should be chosen on a case-by-case basis.

\section{CONCLUSIONS}

In this work, we have directly compared the use of three buckling-based methods (biaxial thermal shrinking, uniaxial thermal shrinking, and SIEBIMM) to measure the elastic moduli of thin films. We conclude that all three methods reliably quantify and yield equivalent values for the elastic moduli of nanobiocomposite thin films, however, thermal shrinking methods consistently give moduli with lower standard deviation between replicate samples. As well, based on sensitivity analyses, thermal shrinking (both uniaxial and biaxial) provides a more robust method of calculating the elastic modulus in comparison to SIEBIMM, which is more sensitive to small deviations in the variables measured. However, all three methods prove useful for mechanical property characterization and each should be chosen on a case-by-case basis depending on equipment and material constraints, as well as the goal of the study.

The buckling-based methods and the analysis presented in this work can be applied to the characterization and evaluation of mechanical performance of both organic and inorganic thin film materials. The three methods compared here are particularly useful for substrate-supported thin films compared to tensile testing and nanoindentation, especially for films composed of soft polymers or nanobiocomposites that cannot easily be made free-standing and/or are highly hygroscopic. Based on this method comparison we suggest that, because of its high precision, low 
sensitivity, and simple experimental setup, biaxial thermal shrinking will find widespread use for high-throughput characterization of thin film mechanical properties.

\section{ASSOCIATED CONTENT}

Supporting Information. A figure of SEM images of wrinkled films (PDF); A table of statistical analysis output data (PDF).

\section{AUTHOR INFORMATION}

\section{Corresponding Author}

*mirabj@mcmaster.ca, (905) 525-9140 x 24507

\section{Author Contributions}

The manuscript was written through contributions of all authors. All authors have given approval to the final version of the manuscript.

\section{Funding Sources}

Natural Sciences and Engineering Research Council of Canada (Discovery Grants RGPIN 201906433and 05252).

\section{ACKNOWLEDGEMENT}

We thank D. Wagner for assistance with the MATLAB algorithm development for Fourier analysis and S. Saem for sputtering of gold films. J.M.M. and E.D.C. are recipients of Early Researcher awards from the Ontario Ministry of Research and Innovation. J.M.M. is the Tier 2 
Canada Research Chair in Micro and Nanostructured Materials. T.C.S is the recipient of a Queen Elizabeth II Ontario Graduate Scholarship. The research presented herein was supported by funding through the Natural Sciences and Engineering Research Council and made use of facilities at the Canadian Centre for Electron Microscopy (CCEM) and the McMaster Centre for Emerging Device Technologies (CEDT).

\begin{abstract}
ABBREVIATIONS
CNC, cellulose nanocrystal; PEI, polyethyleneimine; SIEBIMM, strain induced elastic buckling instability for mechanical measurement; PDMS, polydimethylsiloxane; RH, relative humidity; PS, polystyrene; LbL, layer-by-layer; PAH, poly(allylamine hydrochloride; CNF, cellulose nanofibril; bL, bilayer; SEM, scanning electron microscope; VASE, variable angle spectroscopic ellipsometry; PSD, power spectral density
\end{abstract}

\title{
REFERENCES
}

(1) Stafford, C. M.; Harrison, C.; Beers, K. L.; Karim, A.; Amis, E. J.; Vanlandingham, M. R.; Kim, H. C.; Volksen, W.; Miller, R. D.; Simonyi, E. E. A Buckling-Based Metrology for Measuring the Elastic Moduli of Polymeric Thin Films. Nat. Mater. 2004, 3 (8), 545-550. https://doi.org/10.1038/nmat1175.

(2) Gill, U.; Sutherland, T.; Himbert, S.; Zhu, Y.; Rheinstädter, M. C.; Cranston, E. D.; MoranMirabal, J. M. Beyond Buckling: Humidity-Independent Measurement of the Mechanical Properties of Green Nanobiocomposite Films. Nanoscale 2017, 9 (23), 7781-7790. https://doi.org/10.1039/C7NR00251C. 
(3) Bidhendi, A. J.; Geitmann, A. Methods to Quantify Primary Plant Cell Wall Mechanics. $J$. Exp. Bot. 2019, 70 (14), 3615-3648. https://doi.org/10.1093/jxb/erz281.

(4) Advincula, R. C.; Knoll, W. A Perspective and Introduction to Organic and Polymer Ultrathin Films: Deposition, Nanostructuring, Biological Function, and Surface Analytical Methods. Funct. Polym. Film. 2011, 1, 1-10. https://doi.org/10.1002/9783527638482.ch1.

(5) Vinci, R. P.; Vlassak, J. J. Mechanical Behavior of Thin Films. Annu. Rev. Mater. Sci. 1996, 26, 431-462. https://doi.org/10.1007/978-94-011-3836-9_11.

(6) Chung, J. Y.; Nolte, A. J.; Stafford, C. M. Surface Wrinkling: A Versatile Platform for Measuring Thin-Film Properties. Adv. Mater. 2011, 23 (3), 349-368. https://doi.org/10.1002/adma.201001759.

(7) Stafford, C. M.; Guo, S.; Harrison, C.; Chiang, M. Y. M. Combinatorial and HighThroughput Measurements of the Modulus of Thin Polymer Films Polymer Films. Rev. Sci. Instrum. 2005, 76, 1-5. https://doi.org/10.1063/1.1906085.

(8) Groenewold, J. Wrinkling of Plates Coupled with Soft Elastic Media. Phys. A Stat. Mech. its Appl. 2001, 298 (1-2), 32-45. https://doi.org/10.1016/S0378-4371(01)00209-6.

(9) Chan, E. P.; Kundu, S.; Lin, Q.; Stafford, C. M. Quantifying the Stress Relaxation Modulus of Polymer Thin Films via Thermal Wrinkling. ACS Appl. Mater. Interfaces 2011, 3 (2), 331-338. https://doi.org/10.1021/am100956q.

(10) Nolte, A. J.; Treat, N. D.; Cohen, R. E.; Rubner, M. F. Effect of Relative Humidity on the Young's Modulus of Polyelectrolyte Multilayer Films and Related Nonionic Polymers. Macromolecules 2008, 41 (15), 5793-5798. https://doi.org/10.1021/ma800732j. 
(11) Wilder, E. A.; Guo, S.; Lin-Gibson, S.; Fasolka, M. J.; Stafford, C. M. Measuring the Modulus of Soft Polymer Networks via a Buckling-Based Metrology. Macromolecules 2006, 39 (12), 4138-4143. https://doi.org/10.1021/ma060266b.

(12) Azzam, F.; Chaunier, L.; Moreau, C.; Lourdin, D.; Bertoncini, P.; Cathala, B. Relationship between Young's Modulus and Film Architecture in Cellulose Nanofibril-Based Multilayered Thin Films. Langmuir 2017, $33 \quad$ (17), 4138-4145. https://doi.org/10.1021/acs.langmuir.7b00049.

(13) Eita, M.; Arwin, H.; Granberg, H.; Wågberg, L. Addition of Silica Nanoparticles to Tailor the Mechanical Properties of Nanofibrillated Cellulose Thin Films. J. Colloid Interface Sci. 2011, 363 (2), 566-572. https://doi.org/10.1016/j.jcis.2011.07.085.

(14) Kan, K. H. M.; Cranston, E. D. Mechanical Testing of Thin Film Nanocellulose Composites Using Buckling Mechanics. TAPPI 2013, 12 (4), 9-17.

(15) Cranston, E. D.; Eita, M.; Johansson, E.; Netrval, J.; Salajkov, M.; Arwin, H.; Lars, W. Determination of Young's Modulus for Nanofibrillated Cellulose Multilayer Thin Films Using Buckling Mechanics. Biomacromolecules 2011, 12, 961-969. https://doi.org/10.1021/bm101330w.

(16) Greco, F.; Bellacicca, A.; Gemmi, M.; Cappello, V.; Mattoli, V.; Milani, P. Conducting Shrinkable Nanocomposite Based on Au-Nanoparticle Implanted Plastic Sheet: Tunable Thermally Induced Surface Wrinkling. ACS Appl. Mater. Interfaces 2015, 7, 7060-7065. https://doi.org/10.1021/acsami.5b00825.

(17) Fu, C. C.; Grimes, A.; Long, M.; Ferri, C. G. L.; Rich, B. D.; Ghosh, S.; Ghosh, S.; Lee, L. 
P.; Gopinathan, A.; Khine, M. Tunable Nanowrinkles on Shape Memory Polymer Sheets. Adv. Mater. 2009, 21 (44), 4472-4476. https://doi.org/10.1002/adma.200902294.

(18) De France, K. J.; Babi, M.; Vapaavuori, J.; Hoare, T.; Moran-Mirabal, J.; Cranston, E. D. 2.5D Hierarchical Structuring of Nanocomposite Hydrogel Films Containing Cellulose Nanocrystals. ACS Appl. Mater. Interfaces 2019, 11 (6), 6325-6335. https://doi.org/10.1021/acsami.8b16232.

(19) Greco, F.; Fujie, T.; Ricotti, L.; Taccola, S.; Mazzolai, B.; Mattoli, V. Microwrinkled Conducting Polymer Interface for Anisotropic Multicellular Alignment. ACS Appl. Mater. Interfaces 2013, 5 (3), 573-584. https://doi.org/10.1021/am301908w.

(20) Huang, R.; Stafford, C. M.; Vogt, B. D. Effect of Surface Properties on Wrinkling of Ultrathin Films. J. Aerosp. Eng. 2007, 20 (1), 38-44. https://doi.org/10.1061/(ASCE)08931321(2007)20:1(38).

(21) Hyun, D. C.; Jeong, U. Substrate Thickness: An Effective Control Parameter for Polymer Thin Film Buckling on PDMS Substrates. J. Appl. Polym. Sci. 2010, 112, 2683-2. https://doi.org/10.1002/app.

(22) Hendricks, T. R.; Lee, I. Wrinkle-Free Nanomechanical Film: Control and Prevention of Polymer Film Buckling. Nano Lett. 2007, $7 \quad$ (2), 372-379. https://doi.org/10.1021/n1062544q.

(23) Nolte, A. J.; Cohen, R. E.; Rubner, M. F. A Two-Plate Buckling Technique for Thin Film Modulus Measurements: Applications to Poly Electrolyte Multilayers. Macromolecules 2006, 39, 4841-4847. https://doi.org/10.1021/ma0606298. 
(24) Nolte, A. J.; Rubner, M. F.; Cohen, R. E. Determining the Young's Modulus of Polyelectrolyte Multilayer Films via Stress-Induced Mechanical Buckling Instabilities. Macromolecules 2005, 38, 5367-5370. https://doi.org/10.1021/ma0507950.

(25) Villares, A.; Moreau, C.; Capron, I.; Cathala, B. Chitin Nanocrystal-Xyloglucan Multilayer Thin Films. Biomacromolecules 2014, $15 \quad$ (1), 188-194. https://doi.org/10.1021/bm401474c.

(26) Johansson, E.; Wågberg, L. Tailoring the Mechanical Properties of Starch-Containing Layer-by-Layer Films. Colloids Surfaces A Physicochem. Eng. Asp. 2012, 394, 14-22. https://doi.org/10.1016/j.colsurfa.2011.11.017.

(27) Aulin, C.; Varga, I.; Claesson, P. M.; Wågberg, L.; Lindström, T. Buildup of Polyelectrolyte Multilayers of Polyethyleneimine and Microfibrillated Cellulose Studied by in Situ DualPolarization Interferometry and Quartz Crystal Microbalance with Dissipation. Langmuir 2008, 24 (6), 2509-2518. https://doi.org/10.1021/la7032884.

(28) Moon, R. J.; Martini, A.; Nairn, J.; Simonsen, J.; Youngblood, J. Cellulose Nanomaterials Review: Structure, Properties and Nanocomposites. Chem. Soc. Rev. 2011, 40, 3941-3994. https://doi.org/10.1039/c0cs00108b.

(29) Mariano, M.; Kissi, N. El; Dufresne, A. Cellulose Nanocrystals and Related Nanocomposites: Review of Some Properties and Challenges. J. Polym. Sci. Part B Polym. Phys. 2014, 52 (12), 791-806. https://doi.org/10.1002/polb.23490.

(30) Tashiro, K.; Kobayashi, M. Theoretical Evaluation of Three-Dimensional Elastic Constants of Native and Regenerated Celluloses: Role of Hydrogen Bonds. Polymer (Guildf). 1991, 
$32(8), 1516-1527$.

(31) Lee, K. Y.; Aitomäki, Y.; Berglund, L. A.; Oksman, K.; Bismarck, A. On the Use of Nanocellulose as Reinforcement in Polymer Matrix Composites. Compos. Sci. Technol. 2014, 105, 15-27. https://doi.org/10.1016/j.compscitech.2014.08.032.

(32) Zhang, B.; Zhang, M.; Song, K.; Li, Q.; Cui, T. Shrink Induced Nanostructures for Energy Conversion Efficiency Enhancement in Photovoltaic Devices. Appl. Phys. Lett. 2013, 103 (2). https://doi.org/10.1063/1.4812184.

(33) Kim, J. B.; Kim, P.; Ṕgard, N. C.; Oh, S. J.; Kagan, C. R.; Fleischer, J. W.; Stone, H. A.; Loo, Y. L. Wrinkles and Deep Folds as Photonic Structures in Photovoltaics. Nat. Photonics 2012, 6 (5), 327-332. https://doi.org/10.1038/nphoton.2012.70.

(34) Chen, Z.; Young Kim, Y.; Krishnaswamy, S. Anisotropic Wrinkle Formation on Shape Memory Polymer Substrates. J. Appl. Phys. 2012, $112 \quad$ (12). https://doi.org/10.1063/1.4770483.

(35) Gabardo, C. M.; Zhu, Y.; Soleymani, L.; Moran-Mirabal, J. M. Bench-Top Fabrication of Hierarchically Structured High-Surface-Area Electrodes. Adv. Funct. Mater. 2013, 23 (24), 3030-3039. https://doi.org/10.1002/adfm.201203220.

(36) Kim, H. S.; Crosby, A. J. Solvent-Responsive Surface via Wrinkling Instability. Adv. Mater. 2011, 23 (36), 4188-4192. https://doi.org/10.1002/adma.201101477.

(37) Mechael, S. S.; Wu, Y.; Schlingman, K.; Carmichael, T. B. Stretchable Metal Films. Flex. Print. Electron. 2018, 3, 1-24.

(38) Villares, A.; Moreau, C.; Dammak, A.; Capron, I.; Cathala, B. Kinetic Aspects of the 
Adsorption of Xyloglucan onto Cellulose Nanocrystals: Supporting Information. Soft Matter 2015, 1-4.

(39) Gabardo, C. M.; Hosseini, A.; Soleymani, L. A New Wrinkle in Biosensors: Wrinkled Electrodes Could Be a Breakthrough for Lab-on-a-Chip Devices. IEEE Nanotechnol. Mag. 2016, 6-18.

(40) Pegan, J. D.; Ho, A. Y.; Bachmanc, M.; Khine, M. Flexible Shrink-Induced High Surface Area Electrodes for Electrochemiluminescent Sensing. Lab Chip 2013, 13, 4205-4209. https://doi.org/10.1039/c31c50588j.

(41) Foster, E. J.; Moon, R. J.; Agarwal, U. P.; Bortner, M. J.; Bras, J.; Camarero-Espinosa, S.; Chan, K. J.; Clift, M. J. D.; Cranston, E. D.; Eichhorn, S. J.; et al. Current Characterization Methods for Cellulose Nanomaterials. Chem. Soc. Rev. 2018, 47, 2609-2679. https://doi.org/10.1039/c6cs00895j.

(42) Reid, M. S.; Villalobos, M.; Cranston, E. D. Benchmarking Cellulose Nanocrystals: From the Laboratory to Industrial Production. Langmuir 2017, 33 (7), 1583-1598. https://doi.org/10.1021/acs.langmuir.6b03765.

(43) Canny, J. A Computational Approach to Edge Detection. IEEE Trans. onf Pattern Anal. Mach. Intell. 1986, 8 (6), 697-698. https://doi.org/10.1109/ASICON.2011.6157287.

(44) Stimpson, T. C.; Wagner, D. L.; Cranston, E. D.; Moran-Mirabal, J. M. Image Analysis of Structured Surfaces for Quantitative Topographical Characterization. Submitt. to Nano Sel. 2020.

(45) Mott, P. H.; Dorgan, J. R.; Roland, C. M. The Bulk Modulus and Poisson's Ratio of 
'incompressible" Materials. J. Sound Vib. 2008, 312 (4-5), 572-575. https://doi.org/10.1016/j.jsv.2008.01.026.

(46) Hodge, T. C.; Bidstrup-allen, S. A.; Kohl, P. A. Stresses in Thin Film Metallization. IEEE Trans. Components, Packag. Manuf. Technol. - Part A 1997, 20 (2), 241-250.

(47) Chanliaud, E.; Burrows, K. M.; Jeronimidis, G.; Gidley, M. J. Mechanical Properties of Primary Plant Cell Wall Analogues. Planta 2002, 215 (6), 989-996. https://doi.org/10.1007/s00425-002-0783-8.

(48) Hamby, D. M. A Review of Techniques for Parameter Sensitivity Analysis of Environmental Models. Environ. Monit. Assess. 1994, 32, 135-154.

(49) Martin, C.; Jean, B. Nanocellulose/Polymer Multilayered Thin Films: Tunable Architectures towards Tailored Physical Properties. Nord. Pulp Pap. Res. J. 2014, 29 (1), 19-30. https://doi.org/10.3183/npprj-2014-29-01-p019-030.

(50) Decher, G.; Hong, J.-D. Buildup of Ultrathin Multilayer Films by a Self-Assembly Process, 1 Consecutive Adsorption of Anionic and Cationic Bipolar Amphiphiles on Charged Surfaces. In Makromolekulare Chemie. Macromolecular Symposia; 1991; Vol. 46, pp 321327.

(51) Cranston, E. D.; Gray, D. G.; Rutland, M. W. Direct Surface Force Measurements of Polyelectrolyte Multilayer Films Containing Nanocrystalline Cellulose. Langmuir 2010, 26 (22), 17190-17197. https://doi.org/10.1021/la1030729.

(52) Jean, B.; Dubreuil, F.; Heux, L.; Cousin, F. Structural Details of Cellulose Nanocrystals/Polyelectrolytes Multilayers Probed by Neutron Reflectivity and AFM. 
Langmuir 2008, 24 (7), 3452-3458. https://doi.org/10.1021/la703045f.

(53) Moreau, C.; Beury, N.; Delorme, N.; Cathala, B. Tuning the Architecture of Cellulose Nanocrystal-Poly(Allylamine Hydrochloride) Multilayered Thin Films: Influence of Dipping Parameters. Langmuir 2012, $28 \quad$ (28), 10425-10436. https://doi.org/10.1021/la301293r.

(54) Vanderfleet, O. M.; Reid, M. S.; Bras, J.; Heux, L.; Godoy-Vargas, J.; Panga, M. K. R.; Cranston, E. D. Insight into Thermal Stability of Cellulose Nanocrystals from New Hydrolysis Methods with Acid Blends. Cellulose 2019, 26, 507-528. https://doi.org/10.1007/s10570-018-2175-7.

(55) Weyts, K. F.; Goethals, E. J. New Synthesis of Linear Polyethyleneimine. Polym. Bull. 1988, 19, 13-19.

(56) Roman, F.; Colomer, P.; Calventus, Y.; Hutchinson, J. M. Study of Hyperbranched Poly(Ethyleneimine) Polymers of Different Molecular Weight and Their Interaction with Epoxy Resin. Materials (Basel). 2018, $11 \quad$ (410), 1-26. https://doi.org/10.3390/ma11030410.

(57) Sahiner, N.; Sagbas, S.; Sahiner, M.; Demirci, S. Degradable Tannic Acid/Polyethyleneimine Polyplex Particles with Highly Antioxidant and Antimicrobial Effects. Polym. Degrad. Stab. 2016, 133, 152-161. https://doi.org/10.1016/j.polymdegradstab.2016.08.012.

(58) Kargarzadeh, H.; Mariano, M.; Huang, J.; Lin, N.; Ahmad, I.; Dufresne, A.; Thomas, S. Recent Developments on Nanocellulose Reinforced Polymer Nanocomposites: A Review. 
Polymer (Guildf). 2017, 132, 368-393. https://doi.org/10.1016/j.polymer.2017.09.043.

(59) Ng, H. M.; Sin, L. T.; Bee, S. T.; Tee, T. T.; Rahmat, A. R. Review of Nanocellulose Polymer Composite Characteristics and Challenges. Polym. - Plast. Technol. Eng. 2017, 56 (7), 687-731. https://doi.org/10.1080/03602559.2016.1233277.

(60) Reid, M. S.; Stimpson, T. C.; Niinivaara, E.; Villalobos, M.; Cranston, E. D. Comparing Soft Semicrystalline Polymer Nanocomposites Reinforced with Cellulose Nanocrystals and Fumed Silica. Ind. Eng. Chem. Res. 2018, 57 (1), 220-230. https://doi.org/10.1021/acs.iecr.7b03836.

(61) Xu, X.; Liu, F.; Jiang, L.; Zhu, J. Y.; Haagenson, D.; Wiesenborn, D. P. Cellulose Nanocrystals vs. Cellulose Nanofibrils: A Comparative Study on Their Microstructures and Effects as Polymer Reinforcing Agents. ACS Appl. Mater. Interfaces 2013, 5, 2999-3009. https://doi.org/10.1021/am302624t.

(62) Habibi, Y.; Lucia, L. A.; Rojas, O. J. Cellulose Nanocrystals: Chemistry, Self-Assembly, and Applications. Chem. Rev. 2010, $110 \quad$ (6), 3479-3500. https://doi.org/10.1021/cr900339w.

(63) De France, K. J.; Chan, K. J. W.; Cranston, E. D.; Hoare, T. Enhanced Mechanical Properties in Cellulose Nanocrystal-Poly(Oligoethylene Glycol Methacrylate) Injectable Nanocomposite Hydrogels through Control of Physical and Chemical Cross-Linking. Biomacromolecules 2016, 17 (2), 649-660. https://doi.org/10.1021/acs.biomac.5b01598. 